\title{
"Co-docencia para el aprendizaje de la entrevista médica": un apoyo "in situ" para docentes clínicos en la enseñanza de competencias comunicacionales en pre grado
}

${ }^{1}$ Unidad de Comunicación y Atención en Salud (CREAS). Escuela de

Medicina, Pontificia Universidad Católica de Chile, Santiago de Chile. ${ }^{2}$ Centro de Educación Médica, Pontificia

Universidad Católica de

Chile, Santiago de Chile. ${ }^{3}$ Departamento de Medicina Familiar, Pontificia Universidad Católica de Chile, Santiago de Chile.

aPsicóloga, Magíster en

Salud y Humanización.

bInterno de Medicina,

Escuela de Medicina,

Pontificia Universidad

Católica de Chile, Santiago de Chile.

Fuente de apoyo financiero: Ninguno.

Recibido el 8 de abril de 2011, aceptado el 2 de diciembre de 2011

Correspondencia a: Gricelda Gómez del Río Escuela de Medicina,

Centro de Educación Médica, Pontificia Universidad Católica de Chile.

Alameda 340. Santiago. Chile.

Celular: 93335332

E-mail:ggomez@med. puc.cl

\author{
GRICELDA GÓMEZ ${ }^{1,2, a}$, PHILIPPA MOOREE ${ }^{1,3}$ ESTEBAN ARAOS-BAERISWYL ${ }^{1, \mathrm{~b}}$

\section{Learning doctor-patient communication: does co-teaching help?}

Background: The first part of the medical interview is perhaps one of the most significant components of the doctor's role. How to collect relevant information and how to build a therapeutic relationship with the patient must be taught during the undergraduate curriculum. Clinical teachers have little experience in the teaching skills required to help students learn about doctor-patient communication. Aim: To measure outcomes and perceptions of using a co-teaching model in a course on interviewing during the third year of medical school in the Pontificia Universidad Católica de Chile. Material and Methods: A mixed methods controlled study in which the intervention group participated in a co-teaching workshop with a clinical teacher and a specialist in doctor-patient communication skills (SDPC). The control group participated in a workshop with one clinical teacher. All students completed a questionnaire measuring perception of their learning in communication skills. Semistructured interviews were used to collect the clinical teachers' perception. At the end of the course, the clinical and communication skills of all the students were measured in an objective structured clinical examination. Results: Students and teachers agreed that co-teaching allows greater emphasis and practice in communication skills. The results of the objective structured clinical examination show that despite this greater emphasis, no deleterious effect on the clinical skills was demonstrated during the exam. Conclusions: The use of co-teaching in a course on interviewing allows students to perceive a higher level of learning in communication skills, and possibly enhances their skills. The clinical teachers felt that the co-teacher was an important support.

(Rev Med Chile 2012; 140: 396-403).

Key words: Communication; Physician-Patient Relations; Teaching.
L a entrevista o anamnesis médica es sin duda una de las etapas más significativas del acto médico. En la calidad de esta interacción médico-paciente influyen múltiples factores, los que de forma permanente estarán incidiendo en la relación. Esta etapa de la entrevista no sólo necesita indagar y caracterizar desde la perspectiva del paciente lo que a éste le sucede, sino especialmente crear el vínculo con él y, de esta forma, establecer las bases de la relación profesional. Es en este contexto donde la calidad de la comunicación de ambos participantes resulta crucial.

Si bien comunicación en medicina se considera una competencia clínica básica, ella se diferencia de las demás en varios aspectos ${ }^{1}$. Existe consenso sobre las características que debería tener la docen- 
cia para optimizar el aprendizaje de la comunicación médico-paciente ${ }^{2}$. Docencia en comunicación es más efectiva si se realiza como un complemento al aprendizaje de las habilidades clínicas, en pequeños grupos, aplicando una metodología experiencial ${ }^{3}$. Crear un ambiente seguro de aprendizaje es muy importante. La docencia debe incluir una clara definición de habilidades requeridas para una atención centrada en el paciente y contemplar instancias de observación estructurada de habilidades del estudiante utilizando una guía. Se debe complementar entregando al estudiante un feedback o retroalimentación específica ${ }^{4}$. Docentes clínicos, que realizan docencia en comunicación, requieren de apoyo, muchos de ellos no recibieron estos conceptos en su formación de pre-grado ni están familiarizados con metodología experiencial en pequeños grupos. Una forma de otorgarles apoyo sería usando una metodología de co-docencia. Varios ensayos muestran la eficacia de docencia en comunicación cuando el grupo intervenido participa en talleres con co-docencia ${ }^{5,6}$; esta ha sido propuesta como forma de apoyar el desarrollo académico. Países hispanoparlantes han publicado sobre comunicación, en general ha sido en el ámbito de post-grado ${ }^{7,8}$.

Este artículo describe una experiencia de la Escuela de Medicina de la Pontificia Universidad Católica de Chile en el curso "Entrevista Clínica" introducido el año 2004, primer semestre, tercer año de la carrera. Este curso utiliza variadas metodologías permitiendo entregar a los estudiantes herramientas para investigar y recopilar datos clínicos, también para mejorar sus habilidades comunicacionales (HC) y relacionales. Si bien el equipo de docentes posee amplia experiencia en docencia clínica, es escasa en enseñanza de HC. Por esto se decidió estudiar en los años 2007 y 2008 los resultados y percepciones sobre el apoyo de un especialista en comunicación a los docentes clínicos.

\section{Material y Método}

\section{Población}

Estudiantes de tercer año de medicina fueron divididos aleatoriamente en 14 grupos, de 7 u 8 estudiantes c/u. Cada grupo trabajó quincenalmente en cinco talleres interactivos (espejo y video) con un docente clínico. En estos talleres estudiantes y docentes observaron estructuradamente (con pauta) una entrevista entre estudiante y paciente simulado a través del espejo uni-direccional, A partir de lo observado y de la retroalimentación que entregó el paciente simulado, se analizó y reflexionó sobre aspectos clínicos y comunicacionales, En esta actividad participaron 7 docentes clínicos con dos grupos de estudiantes cada uno.

\section{Consentimiento informado}

El profesor jefe solicitó verbalmente el consentimiento de participar en el estudio, a estudiantes y docentes.

\section{Intervención}

Cada grupo intervenido, realizó co-docencia con un docente clínico y un no-clínico, especialista en comunicación (EC).La EC, licenciada en relaciones humanas y familia, formada en el enfoque centrado en la persona con 15 años de experiencia en metodología experiencial en pequeños grupos en la Escuela de Medicina de la Universidad Católica. Objetivos, metodología y tiempo de los talleres fueron iguales en ambos grupos (intervenidos y controles).

Diferencias en la docencia en: grupos intervenidos del grupo control:

Presencia de EC quien facilitó el feedback a los estudiantes sobre su competencia comunicacional, describiéndole aquellas habilidades que favorecían u obstaculizaban la relación.

1. Los estudiantes conocieron, practicaron y recibieron feedback sobre HC usando una adaptación de la pauta original que presentaba 9 descriptores de competencias comunicacionales, fue modificada considerando los objetivos del curso, nivel de formación de los estudiantes y teniendo como marco de referencia la Guía de Calgary Cambridge ${ }^{9}$. Se detalló cada uno de los 9 descriptores dando como resultado una guía de 27 ítems de $\mathrm{HC}$ que fueron agrupadas en relación con tres áreas de la entrevista: Inicio de la entrevista, Recogiendo información y Atención centrada en el paciente; cada área correspondía a una sesión de aprendizaje. El formato original de respuesta SI/NO fue cambiado a un formato abierto que permitió escribir comentarios sobre cada ítem.

2. Al final de cada sesión los co-docentes reflexionaron sobre: dinámica del grupo, comunicación entre estudiantes durante el taller, y avances en su aprendizaje. 


\section{Mediciones}

Al finalizar el semestre se evaluó:

1. Percepción de los estudiantes sobre:

a. Aprendizaje de HC, con encuesta auto-aplicada, anónima, repartida a todos los estudiantes del curso, con un formato SI/NO, si conoció, practicó y recibió feedback sobre los 27 ítems de HC separada en tres áreas: Iniciando la entrevista (6), Recogiendo información (13), Atención centrada en el paciente (8) (Tabla 1) dando un total de 81 percepciones por estudiante.

b. Co-docencia, con encuesta auto-aplicada y anónima a los intervenidos.

\section{Tabla 1. Habilidades comunicacionales evaluadas como percepción}

\begin{tabular}{|c|c|c|c|}
\hline & & Conocí Practiqué & $\begin{array}{c}\text { Recibí } \\
\text { feedback }\end{array}$ \\
\hline \multicolumn{4}{|c|}{ Iniciando la entrevista } \\
\hline 1 & Saludar al paciente por su nombre & & \\
\hline 2 & Estar atento al confort físico del paciente, indicarle dónde sentarse & & \\
\hline 3 & Presentarme al paciente diciendo su nombre & & \\
\hline 4 & Describir mi rol de estudiante de tercer año & & \\
\hline 5 & Explicar al paciente que el tutor me supervisará & & \\
\hline 6 & Informar al paciente que le realizaré algunas preguntas & & \\
\hline 7 & Hacer preguntas al paciente manteniendo contacto visual & & \\
\hline 8 & $\begin{array}{l}\text { Comenzar la entrevista con una pregunta abierta referida al motivo de consulta } \\
\text { antes de obtener la información demográfica }\end{array}$ & & \\
\hline 9 & Permitir al paciente relatar el motivo de consulta sin interrupción precoz & & \\
\hline 10 & $\begin{array}{l}\text { Explicar al paciente que le preguntaré algunos datos que no están referidos al } \\
\text { motivo de consulta (datos demográficos) }\end{array}$ & & \\
\hline 11 & Reconfirmar el motivo de consulta con un resumen & & \\
\hline 12 & Utilizar de manera atingente preguntas abiertas y cerradas & & \\
\hline \multicolumn{4}{|c|}{ Recogiendo la información } \\
\hline 13 & $\begin{array}{l}\text { Escuchar activamente permitiendo al paciente completar las explicaciones sin } \\
\text { interrupciones }\end{array}$ & & \\
\hline 14 & Aceptar que el paciente hable a su ritmo sin terminarle las frases & & \\
\hline 15 & $\begin{array}{l}\text { Facilitar en forma verbal las respuestas del paciente (continuadores, parafraseo, } \\
\text { repeticiones) }\end{array}$ & & \\
\hline 16 & $\begin{array}{l}\text { Facilitar en forma no verbal las respuestas del paciente (silencios, asentimiento } \\
\text { con la cabeza, etc.) }\end{array}$ & & \\
\hline 17 & $\begin{array}{l}\text { Verificar mi comprensión haciendo pequeños resúmenes de lo que el paciente } \\
\text { ha descrito }\end{array}$ & & \\
\hline 18 & Chequear con el paciente cuando yo no entendí algo & & \\
\hline 19 & Explorar si el paciente tiene otros problemas & & \\
\hline 20 & Usar lenguaje adecuado al paciente, sin tecnicismos & & \\
\hline \multicolumn{4}{|c|}{ Atención centrada en el paciente } \\
\hline 21 & Usar lenguaje adecuado, sin tecnicismos & & \\
\hline 22 & Captar señales verbales y no verbales del paciente & & \\
\hline 23 & Reconocer las emociones expresadas & & \\
\hline 24 & Verbalizar las emociones que reconozco en el paciente & & \\
\hline 25 & Indagar las creencias del paciente relativas al motivo de consulta & & \\
\hline 26 & Indagar los temores del paciente respecto al motivo de consulta & & \\
\hline 27 & Indagar expectativas del paciente relativas al motivo de consulta & & \\
\hline
\end{tabular}


2. Percepción de docentes clínicos sobre metodología de co-docencia utilizando entrevistas semi-estructuradas según guión temático, aplicada por un estudiante-ayudante. Estas fueron grabadas, transcritas textualmente y luego analizadas.

3. Competencias de los estudiantes. Al final del semestre de 2007 se programó un examen clínico objetivo estructurado (ECOE/OSCE) de 7 estaciones para evaluar habilidades y competencias clínicas en medicina, para ser rendido por todos los estudiantes. Se consideró en nuestro análisis, la nota final del ECOE y los puntajes de cuatro estaciones: dos estaciones clínicas (A1 "hemorragia" y B1 "síndrome febril") y dos estaciones referente a la redacción de historias clínicas (A2 y B2). En ambas estaciones clínicas el estudiante realizó una entrevista con un paciente estandarizado, se midió el logro/no-logro de 18 ítems de habilidades clínicas y de 9 ítems de HC (Tabla 2), dando un puntaje total a la estación. La nota final del ECOE fue construida con los puntajes de todas las estaciones.

\section{Análisis}

Los grupos y subgrupos de análisis están descritos en Figura 1 y 2. Al analizar las diferencias de percepción de los estudiantes se utilizó el test $\chi^{2}$, considerándose significativo un valor $\mathrm{p}<0,05$. El puntaje obtenido en las cuatro estaciones de ECOE como nota final, se comparo entre grupo intervenido y control mediante test $t$ de Student

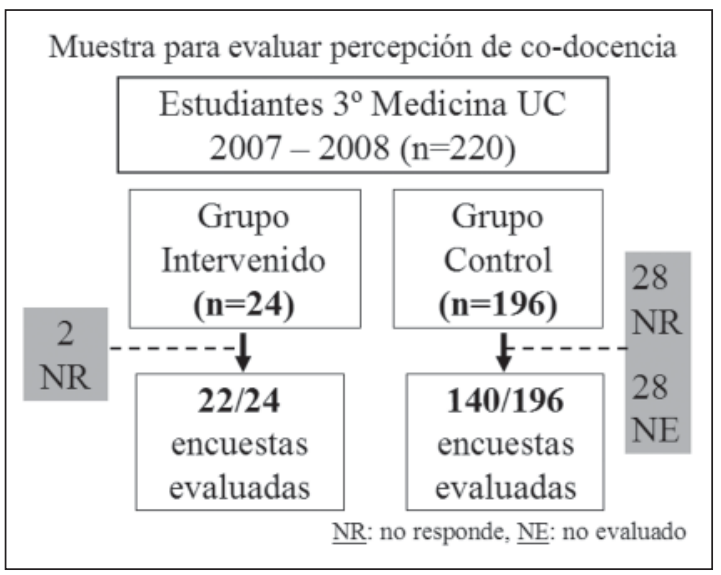

Figura 1. Selección de estudiantes para evaluar percepción de metodología.
Tabla 2. Ítems de Habilidades Comunicacionales medidos en 2 estaciones del ECOE/OSCE

\begin{tabular}{|c|c|c|}
\hline 1 & \multirow{8}{*}{ 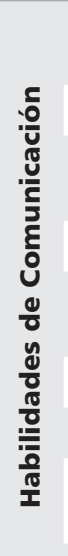 } & $\begin{array}{l}\text { Saluda al paciente (lo invita a tomar asiento y } \\
\text { ponerse cómodo) }\end{array}$ \\
\hline 2 & & Se presenta \\
\hline 3 & & $\begin{array}{l}\text { Mira a los ojos al paciente durante la entre- } \\
\text { vista }\end{array}$ \\
\hline 4 & & Utiliza al menos dos preguntas abiertas \\
\hline 5 & & $\begin{array}{l}\text { Investiga aspectos psicológicos y/o afectivos } \\
\text { del paciente (temores) }\end{array}$ \\
\hline 6 & & Utiliza un lenguaje simple, sin tecnicismos \\
\hline 7 & & Investiga datos demográficos del paciente \\
\hline 8 & & $\begin{array}{l}\text { Permite al paciente relatar sus síntomas sin } \\
\text { interrupción precoz (antes de } 30 \text { seg) }\end{array}$ \\
\hline 9 & & Pregunta por el motivo de consulta \\
\hline
\end{tabular}

para muestras independientes. Los subgrupos fueron comparados con la prueba no paramétrica de U Mann-Whitney. Para el análisis se utilizó el programa estadístico Minitab 15.

\section{Resultados}

\section{Percepción de los estudiantes}

Se entregó una encuesta auto aplicada al 100\% de los estudiantes del curso en 2007 y 2008, $87 \%$ $(192 / 220)$ de encuestas fueron respondidas, 28 encuestas fueron declaradas inválidas (incom-

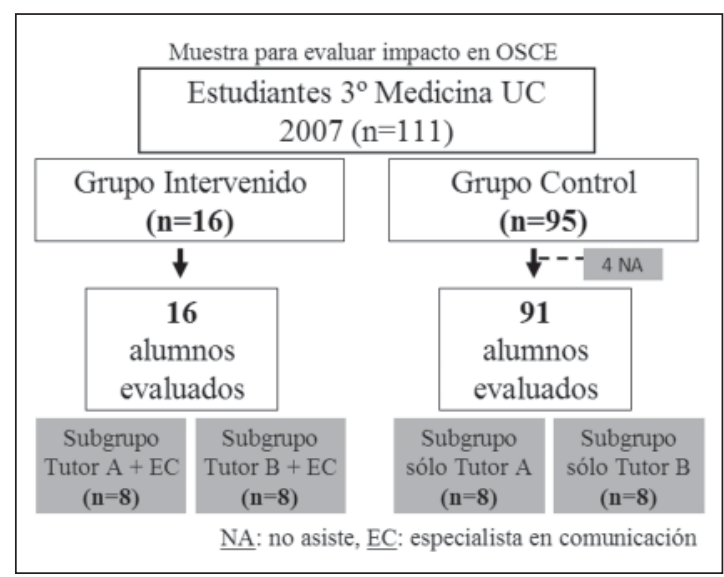

Figura 2. Selección de estudiantes para evaluar impacto en ECOE/OSCE. 


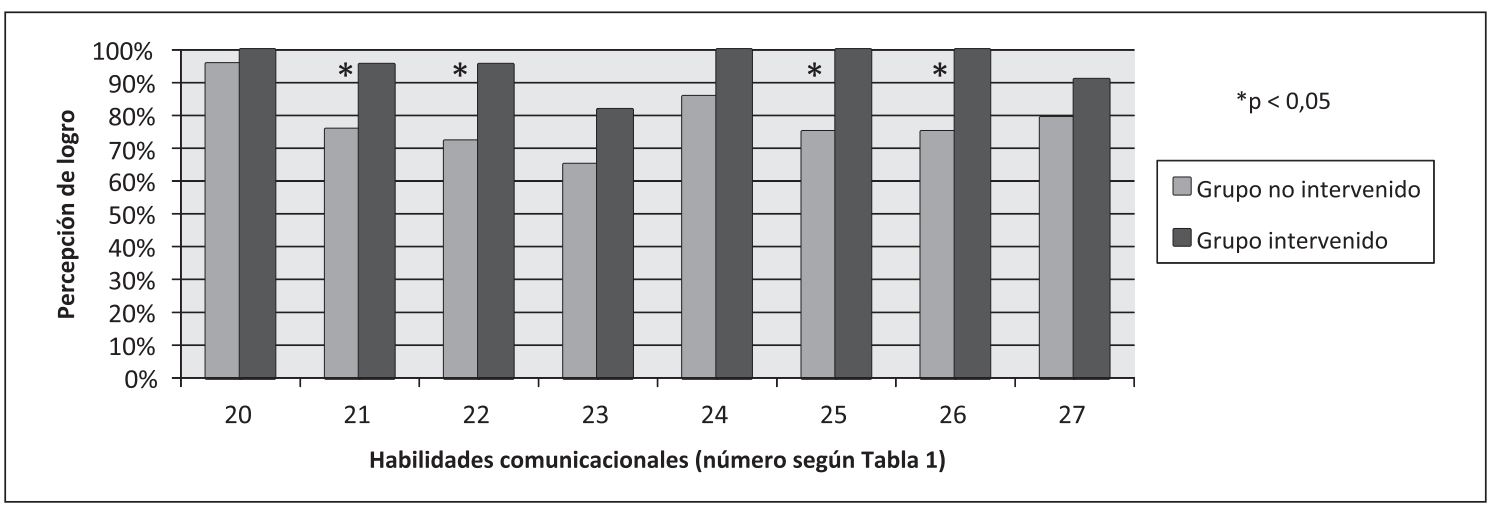

Figura 3. Gráfico de percepción de haber recibido feedback en el área "Atención centrada en el paciente".

pletas o no legibles) por tanto, no analizadas. Se analizó el 91,7\% (22/24) de encuestas aplicadas al grupo intervenido y el 71\% (140/196) del grupo no intervenido. Grupo intervenido percibió que, comparado con grupo control, había conocido, practicado y recibido feedback en mayor porcentaje en todos los ítems de $\mathrm{HC}$, con excepción de un ítem; sin embargo, esta diferencia fue estadísticamente significativa solamente en 14 de los 81 ítems (19\%). Estos son, 4 ítems en el área "Iniciando la entrevista", 3 ítems en el área "Recogiendo la información" y 7 ítems en el área de "Atención centrada en el paciente. La Figura 3 muestra diferencias en la percepción de haber recibido feedback entre grupo intervenido y control en el área "Atención centrada en el paciente".

\section{Percepción de los docentes clínicos}

Se entrevistaron 5 de 7 docentes clínicos (72\%). Todos (con excepción de uno que no opinó sin haber vivido la experiencia) expresaron adhesión a la propuesta de co-docencia en tercer año con un EC. Consideraron que podría complementar la formación clínica del estudiante, quien está comenzando su aprendizaje de entrevista. Declararon que "son dos experiencias que se juntan y al finy al cabo son enriquecedoras para los estudiantes". Los entrevistados reconocieron falta de formación en comunicación, indicando que su docencia es más bien intuitiva: "si bien es cierto yo tengo la formación como clínico, en comunicación está bastante en bruto" "sumamente positivo, una dupla donde hay un clínico y un especialista en comunicación aporta a la discusión de lo que uno no sabe, sobre todo en entender que la comunicación no es un arte, es una ciencia que tiene sus principios y su nomenclatura".
Los docentes participantes en co-docencia identificaron mayor profundización en elementos comunicacionales en los grupos intervenidos. "Desde mi punto de vista, yo como docente creo que en el grupo en que ella estuvo-tuve un grupo con EC y uno sin ella-y creo que los estudiantes que estuvieron con ella y conmigo, debieran haber aprendido muchas más técnicas y haber logrado habilidades comunicativas que el grupo sin co-docencia".

Visualizaron dificultades en la organización al masificar esta metodología, entendiendo que habría que duplicar el número de docentes. Demuestran preocupación por el poco tiempo que se dispone para cada sesión. "Me parece que está muy comprimido el tiempo. Habría que ver cómo hacerlo".

\section{Demostración de competencias en ECOE}

Cuatro estudiantes del grupo control no asistieron a la evaluación; se analizaron los resultados de $100 \%$ del grupo intervenido (16/16) y el $95,8 \%$ de los resultados del grupo control (91/95). Los resultados de 4 estaciones analizadas están resumidos en Figura 4. En las estaciones A1 y B1, el grupo intervenido logró un mejor puntaje promedio que el grupo control. En la estación B1, las diferencias fueron estadísticamente significativas (grupo intervenido 95,4 $\pm 4,9$, y grupo control $86,8 \pm 10,1, p<0,0001)$. En la estación A2 no hubo diferencias entre los 2 grupos, no obstante en la estación B2, el grupo intervenido obtuvo un puntaje significativamente mejor que el grupo control $(p<0,05)$. En estaciones A1 y B1 los evaluadores identificaron como logrado la mayoría de los 9 ítems relacionados con $\mathrm{HC}$ en $>90 \%$ de estudiantes de ambos grupos, con la excepción del ítem 
EDUCACIÓN MÉDICA

Co-docencia como metodología para el aprendizaje/enseñanza de la entrevista médica - G. Gómez et al

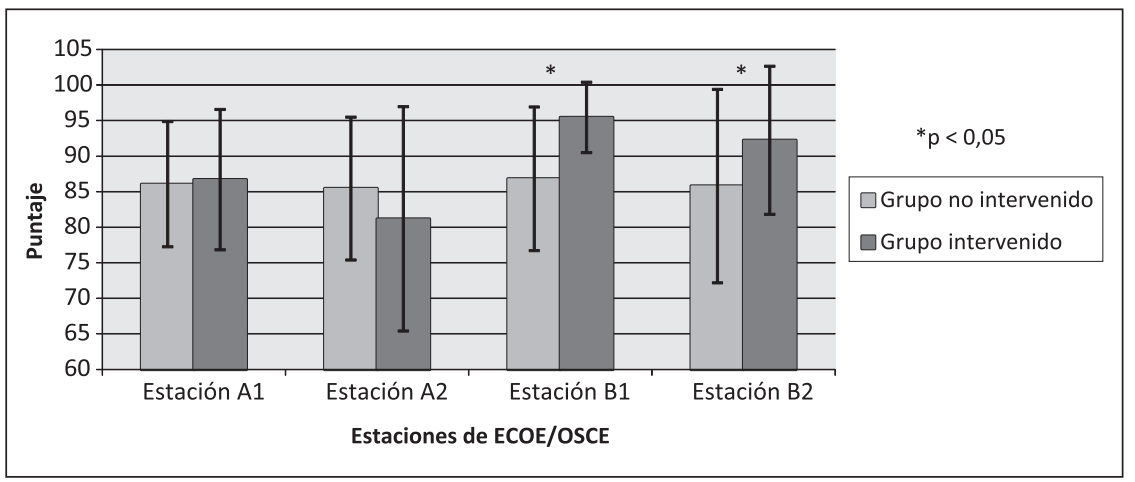

Figura 4. Gráfico del puntaje obtenido en ECOE/OSCE.
"Investigar aspectos psicológicos y/o afectivos del paciente, temores" que fue logrado por solamente $78 \%$ de los estudiantes. La nota final del ECOE del grupo intervenido fue significativamente superior a la del grupo control: 6,49 $\pm 0,295$ contra 6,29 \pm $0,35(\mathrm{p}<0,05)$.

Al evaluar los subgrupos, se observó la tendencia de que ambos subgrupos intervenidos obtienen mejor puntajes en las estaciones B1 y B2, además de mejorar su nota final en ECOE, sin significancia estadística (Tabla 3).

\section{Discusión}

Se reconocen las dificultades que tienen docentes clínicos en la enseñanza de HC, hay un llamado a apoyarlos ${ }^{2,10}$. No se encontraron otros estudios realizados en Chile sobre cómo apoyar in-situ a los docentes clínicos en el desafío de realizar docencia clínica y comunicacional en forma integrada.

Nuestros estudiantes y docentes concuerdan en que la metodología de co-docencia (clínico y EC) permite se dé mayor énfasis y se pongan en práctica las HC. El EC fue visto como un complemento que apoya y permite cumplir mejor con los objetivos del taller. "El especialista en comunicación está sensibilizado para identificar habilidades comunicacionales, lo verbal, no verbal, gestual, relacional, emociones, lo que permite conocer mejor al paciente". Realizar co docencia requiere de mayores recursos económicos como humanos lo que dificulta la incorporación de un EC. Lo recomendable sería la capacitación formal de todos los docentes clínicos del curso "Entrevista Médica" por ser el inicio de la formación de los estudiantes en la relación médico-paciente. Algunas escuelas de medicina ya incluyen este tipo de capacitación en la formación
Tabla 3. Nota final de ECOE/OSCE por subgrupo

\begin{tabular}{|lc|}
\hline Subgrupo & $\begin{array}{c}\text { Nota final ECOE } \pm \\
\text { desviación estándar }\end{array}$ \\
\hline Subgrupo Tutor A & $6,295 \pm 0,279$ \\
\hline Subgrupo Tutor A + EC & $6,503 \pm 0,233$ \\
\hline Subgrupo Tutor B & $6,436 \pm 0,182$ \\
\hline Subgrupo Tutor B + EC & $6,4824 \pm 0,363$ \\
\hline
\end{tabular}

EC: especialista en comunicación.

de sus docentes ${ }^{11}$. En la Pontificia Universidad Católica de Chile, en el marco del Diplomado de Educación Médica, se han formado 250 docentes en "Introducción a la Docencia en Comunicación Centrada en la Persona"12; sin embargo, persiste la necesidad de apoyo "en terreno", es en este escenario donde cobra relevancia el aporte de un co-docente EC.

Los resultados de la evaluación de competencias en el ECOE demuestran que, a pesar del mayor énfasis en la relación médico-paciente en los grupos intervenidos, no perjudicó el aprendizaje de las competencias clínicas. No obstante lo anterior, el cumplimiento de las HC era altísimo en ambos grupos y no hubo discriminación entre los estudiantes. Este es un fenómeno reconocido en otros estudios ${ }^{13,14}$. Ha sido cuestionado si los ECOE son el mejor instrumento para evaluar HC. En efecto, lo anterior presenta algunas dificultades como limitación del tiempo en el contexto de una evaluación sumativa y la necesidad de contar con evaluadores capacitados en $\mathrm{HC}^{15}$. Se requiere un alto número de estaciones para lograr confiabilidad ${ }^{16}$. En este caso hubo sólo dos estaciones de comunicación de 15 minutos cada una. El checklist 
utilizado en el ECOE, en formato Si/No, no ha sido validado aún, y fue completado por los mismos docentes que realizaron el curso, los que a su vez comentaron en las entrevistas que no tienen formación en comunicación ("Los docentes nunca tuvimos entrenamiento formal en comunicación"; "yo tengo la experiencia clínica y experiencia en comunicación en bruto"). Es posible que los docentes no hayan logrado discriminar los estudiantes que usaron $\mathrm{HC}$ efectivas en las estaciones al usar este tipo de pauta polarizada. El estudiante sabiendo que sus habilidades serán evaluadas con un checklist $\mathrm{Si} / \mathrm{No}$, privilegia preguntas cerradas, asumiendo que así recolectará más información en menos tiempo, siendo contradictorio con lo aprendido, que privilegia preguntas abiertas al inicio de la entrevista para permitir escuchar la perspectiva del paciente. La evaluación muchas veces es la fuerza que dirige el aprendizaje del estudiante, esta forma de evaluación puede contribuir a la dificultad de demostrar sus HC.

Cabe destacar que en este estudio no se incluyó una pauta evaluativa para ser completada por el paciente simulado. El punto de vista del paciente aumenta la validez de la evaluación de $\mathrm{HC}^{17}$. La evaluación realizada por pacientes simulados generalmente representa la perspectiva de pacientes reales ${ }^{18}$, se ha demostrado que pueden completar pautas de cotejo en forma confiable y precisa ${ }^{19}$. En las escuelas de medicina en Estados Unidos de Norteamérica en $95 \%$ de ECOE son los pacientes quienes completan las pautas de cotejo ${ }^{20}$.

Centrarse en la agenda del paciente es crucial en una entrevista efectiva, mejora la satisfacción del paciente ${ }^{21}$. Esta área (Atención centrada en el paciente) fue la peor lograda en el ECOE en ambos grupos (ítem "Investigar aspectos psicológicos y/o afectivos del paciente, temores)". Sin embargo, un número significativamente mayor de estudiantes del grupo intervenido percibía haber aprendido varios de los ítems de esta área "Captar las señales verbales/no verbales en el paciente" y "reconocer emociones expresadas por los pacientes", a pesar que se enseñó en la penúltima sesión de aprendizaje y por tanto, menos tiempo de práctica. Pareciera que co-docencia facilita el aprendizaje de este tema, que es particularmente difícil para un estudiante de tercer año de medicina.

Una debilidad en la evaluación cuantitativa de este estudio fue el bajo número de estudiantes en el grupo intervenido (24) comparado con el grupo control (196). Éste pudiera ser el motivo por el que no se logró demostrar mayor diferencia estadísticamente significativa en las encuestas de percepción y el desempeño en el ECOE.

$\mathrm{Si}$ queremos enseñar a nuestros estudiantes habilidades que les permitan realizar entrevistas efectivas y centradas en el paciente, tenemos que seguir investigando cuál es la mejor forma de apoyar a los docentes clínicos. Es clave capacitarlos para que puedan modelar, enseñar y evaluar $\mathrm{HC}^{22}$. La co-docencia con un EC es una forma muy aceptable de apoyar a los docentes "en terreno", y puede ser complementaria a la capacitación de éstos ${ }^{23}$. Debemos estar atentos al estilo de evaluación que usamos y asegurar que el mensaje enviado a los estudiantes sea claro: para ser un buen médico las competencias clínicas como las comunicacionales son importantes.

Agradecimientos: Quisiéramos agradecer a: Los estudiantes de tercer año de medicina 2007/2008 que participaron en el estudio y los docentes de tercer año que fueron entrevistados y especialmente a los docentes que aceptaron participar en co-docencia.

\section{Referencias}

1. Moore P, Gómez G, Kurtz S. Comunicación médico-paciente: Una de las competencias básicas pero diferentes. Aten Primaria. In press 2011.

2. Makoul G, Schofield T. Communication teaching and assessment in medical education: an international consensus statement. Netherlands Institute of Primary Health Care. Patient Educ Couns 1999; 37 (2): 191-5.

3. Moore P, Gómez G. Comunicarse efectivamente en Medicina ¿Cómo adquirir Habilidades Comunicacionales durante la carrera de Medicina? Ars Medica 2007; 15: 171-81.

4. Kurtz S, Silverman J, Draper J. Teaching and learning Communication Skills in Medicine. Radcliffe Medical Press; 2005.

5. Kruijver IPM, Kerkstra A, Kerssens JJ, Holtkamp CCM, Bensing JM, HBM. Communication between nurses and simulated patients with cancer: evaluation of a communication training programme... including commentary by Heaven C and Payne S. European Journal of Oncology Nursing 2001; 5(3): 140-53.

6. Stewart M, Brown JB, Hammerton J, Donner A, Gavin A, Holliday RL, et al. Improving communication between 
doctors and breast cancer patients. Annals of family medicine 2007; 5: 387-94.

7. Ruiz-Moral R. Programas de formación en comunicación clínica: una revisión de su eficacia en el contexto de la enseñanza médica. Educación Médica 2003; 6(4): 159-67.

8. Ruiz-Moral R, Perula de Torres LA, Barrios L, ParrasRejano JM, Aparicio J, Lemos A, et al. Efectividad de un curso de pregrado sobre medicina de familia. Validez y fiabilidad de un test de concordancia script. Educación Médica 2004; 7 (2): 78-84.

9. Kurtz SM. Doctor-patient communication: principles and practices. Can J Neurol Sci 2002; 29 Suppl 2: S23-9.

10. Makoul G. Essential elements of communication in medical encounters: the Kalamazoo consensus statement. Acad Med 2001; 76(4): 390-3.

11. Bylund CL, Brown RF, di Ciccone BL, Levin TT, Gueguen JA, Hill C, et al. Training faculty to facilitate communication skills training: development and evaluation of a workshop. Patient Educ Couns 2008; 70 (3): 430-6.

12. Triviño X, Sirhan M, Moore P, Montero L. Impacto de un programa de formación en docencia en una escuela de medicina. 2011. Rev Med Chile 2011; 139: 1508-15.

13. Epstein RM. Assessment in medical education. N Engl J Med 2007; 356 (4): 387-96.

14. Ruiz-Moral R, Perula de Torres LA. [Validity and reliability of a tool for assessing clinical communication in consultations: the CICAA questionnaire]. Aten Primaria 2006; 37 (6): 320-4.
15. Duffy FD, Gordon GH, Whelan G, Cole-Kelly K, Frankel $\mathrm{R}$, Buffone $\mathrm{N}$, et al. Assessing competence in communication and interpersonal skills: the Kalamazoo II report. Acad Med 2004; 79 (6): 495-507.

16. Epstein RM, Hundert EM. Defining and assessing professional competence. JAMA 2002; 287 (2): 226-35.

17. Schirmer JM, Mauksch L, Lang F, Marvel MK, Zoppi K, Epstein RM, et al. Assessing communication competence: a review of current tools. Fam Med 2005; 37 (3): 184-92.

18. Sanson-Fisher R, Maguire P. Should skills in communicating with patients be taught in medical schools? Lancet 1980; 2 (8193): 523-6.

19. De Champlain AF, Margolis MJ, King A, Klass DJ. Standardized patients' accuracy in recording examinees' behaviors using checklists. Acad Med 1997; 72 (10 Suppl 1): S85-7.

20. Hauer KE, Hodgson CS, Kerr KM, Teherani A, Irby DM. A national study of medical student clinical skills assessment. Acad Med 2005; 80 (10 Suppl): S25-9.

21. Moore P, Gómez G, Kurtz S, Vargas A. [Doctor patient communication: which skills are effective?]. Rev Med Chile 2010; 138 (8): 1047-54.

22. Kalet A, Pugnaire MP, Cole-Kelly K, Janicik R, Ferrara E, Schwartz MD, et al. Teaching communication in clinical clerkships: models from the macy initiative in health communications. Acad Med 2004; 79 (6): 511-20.

23. Triviño X, Sirhan M, Moore P, Reyes C. [Faculty development for clinical teachers in medicine]. Rev Med Chile 2009; 137 (11): 1516-22. 